\title{
Next generation sequencing of exceptional responders with BRAF-mutant melanoma: implications for sensitivity and resistance
}

Jennifer Wheler ${ }^{1 *}$, Roman Yelensky², Gerald Falchook¹, Kevin B Kim³ ${ }^{3}$ Patrick Hwu ${ }^{3}$, Apostolia M Tsimberidou', Philip J Stephens ${ }^{2}$, David Hong ${ }^{1}$, Maureen T Cronin $^{2}$ and Razelle Kurzrock ${ }^{4}$

\begin{abstract}
Background: Patients with BRAF mutation-positive advanced melanoma respond well to matched therapy with BRAF or MEK inhibitors, but often quickly develop resistance.

Methods: Tumor tissue from ten patients with advanced BRAF mutation-positive melanoma who achieved partial response (PR) or complete response (CR) on BRAF and/or MEK inhibitors was analyzed using next generation sequencing (NGS) assay. Genomic libraries were captured for 3230 exons in 182 cancer-related genes plus 37 introns from 14 genes often rearranged in cancer and sequenced to average median depth of $734 \mathrm{X}$ with $99 \%$ of bases covered $>100 X$.

Results: Three of the ten patients (median number of prior therapies $=2$ ) attained prolonged CR (duration $=23.6+$ to 28.7+ months); seven patients achieved either a PR or a short-lived CR. One patient who achieved CR ongoing at 28.7+ months and had tissue available close to the time of initiating BRAF inhibitor therapy had only a BRAF mutation. Abnormalities in addition to BRAF mutation found in other patients included: mutations in NRAS, APC and NF1; amplifications in BRAF, aurora kinase A, MYC, MITF and MET; deletions in CDKN2A/B and PAX5; and, alterations in RB1 and ATM. Heterogeneity between patients and molecular evolution within patients was noted.

Conclusion: NGS identified potentially actionable DNA alterations that could account for resistance in patients with BRAF mutation-positive advanced melanoma who achieved a PR or CR but whose tumors later progressed. A subset of patients with advanced melanoma may harbor only a BRAF mutation and achieve a durable CR on BRAF pathway inhibitors.
\end{abstract}

Keywords: BRAF mutation, Melanoma, Next generation sequencing, Resistance, Time to treatment failure

\section{Background}

Over $50 \%$ of melanomas are characterized by the presence of a BRAF mutation [1]. The most common BRAF mutation (BRAF V600E) leads to constitutive activation of the mitogen-activated protein kinase (MAPK) pathway. Targeting BRAF with RAF-selective inhibitors has demonstrated remarkable tumor shrinkage in those tumors with BRAF mutations [2-4]. Despite these remarkable

\footnotetext{
* Correspondence: jjwheler@mdanderson.org

'Department of Investigational Cancer Therapeutics - a Phase I Clinical Trials Program, Unit 455, The University of Texas MD Anderson Cancer Center, 1515 Holcombe Blvd, Houston, TX 77030, USA

Full list of author information is available at the end of the article
}

results, response to BRAF inhibitors is transient for most patients with advanced melanoma.

Previous pre-clinical studies have shown that retreatment with a second BRAF inhibitor in cells that have become resistant to another BRAF inhibitor is unlikely to be an effective strategy [5]. Nor are secondary BRAF mutations believed to play a large role after development of resistance [6,7]. However, reactivation of MAPK pathway through various mechanisms may be in part responsible for the development of acquired resistance [6]. Combination strategies may help to overcome resistance. For example, combining a BRAF inhibitor with agents that target insulin-like growth factor 1 receptor (IGF1R), downstream phosphatidylinositol 3-kinase (PI3K)/AKT 
signaling and/or MEK pathways may serve to enhance therapeutic effects [5,8]. Identification of molecular alterations in addition to $B R A F$ may help explain why resistance develops more quickly in some patients, and suggest rationale strategies to overcome resistance.

In this pilot study, we investigated patients with advanced melanoma who were responders on clinical trials using BRAF and/or MEK inhibitors.

\section{Methods}

\section{Patients}

Patients with BRAF-mutant, advanced melanoma who experienced treatment failure with standard therapy, and subsequent partial response (PR) or complete response (CR) on BRAF, MEK, and BRAF/MEK combination targeted trials, and who had tissue available for molecular analysis were eligible. The study was carried out by collaboration between the Department of Investigational Cancer Therapeutics (Phase I Clinical Trials Program) at The University of Texas MD Anderson Cancer Center (MD Anderson) and Foundation Medicine (Boston, MA). The registration of patients in the database, pathology assessment, and preliminary limited mutation analysis (see below) were performed at MD Anderson. Subsequent molecular evaluation with NGS was performed at Foundation Medicine. The study was reviewed and approved by the MD Anderson Institutional Review Board (IRB 5 IRB00006023) with a waiver of authorization to use and disclose protected health information. All patients consented for experimental therapeutic interventions according to institutional guidelines, and all patients had consented to anonymized assessments and analysis of data and outcome of therapy.

\section{Tissue samples and molecular analyses}

Mutation analysis at MD Anderson: Archival formalinfixed, paraffin-embedded tissue blocks or material from fine-needle aspiration biopsy obtained from diagnostic and/or therapeutic procedures was used to test for $B R A F$ mutations. BRAF mutation testing was performed in the CLIA-certified Molecular Diagnostic Laboratory within the Division of Pathology and Laboratory Medicine at MD Anderson. DNA was extracted from micro-dissected paraffin-embedded tumor sections and analyzed using a polymerase chain reaction (PCR)-based DNA sequencing method for BRAF codons 595-600 mutations of exon 15 by pyrosequencing as previously described [9]. Whenever possible, testing for other mutations such as Kirsten rat sarcoma viral oncogene homolog $(K R A S)$ and neuroblastoma rat sarcoma viral oncogene homolog (NRAS), PIK3CA [10], and TP53 was performed. Phosphatase and tensin homolog (PTEN) deletion was assessed using immunohistochemistry and the DAKO antibody (Carpinteria, Ca.) [11].
NGS analysis at Foundation Medicine: Genomic libraries were captured for 3230 exons in 182 cancer-related genes plus 37 introns from 14 genes often rearranged in cancer and sequenced to average median depth of $734 \mathrm{X}$ with $99 \%$ of bases covered $>100 \mathrm{X}$ [12] (Additional file 1). The molecular alterations were reported as somatic alterations of known significance and somatic alterations of unclear significance based on the impact of these molecular alterations on tumorigenesis as stated in the scientific literature.

\section{Treatment and evaluation}

Starting in July 2010, consecutive patients with melanoma and available tissue who achieved a PR or CR while on a BRAF and/or MEK inhibitor were studied. Treatment continued until disease progression or unacceptable toxicity occurred.

Assessments were performed as specified in each protocol at the beginning of each new treatment cycle. Efficacy was assessed using computed tomography and/or positron emission tomography scan at baseline and then every two cycles (eight weeks). All radiographs were read in the Department of Radiology at MD Anderson. Responses were categorized per Response Evaluation Criteria in Solid Tumors (RECIST) version 1.0 or 1.1 depending on the study the patient was enrolled in (Additional file 2) and were reported as best response $[13,14]$.

\section{Statistical analysis}

This is a pilot study with descriptive analyses used to summarize patient characteristics. Time to treatment failure (TTF) was defined as the time interval from the start of therapy to the first observation of disease progression per RECIST version 1.0 or 1.1 depending on the study, or death, or removal from study for any reason.

\section{Results \\ Patients}

A total of ten patients with $B R A F$-mutant, advanced melanoma who had achieved a PR or CR on a BRAF and/or MEK targeted drug were analyzed for molecular alterations. The median age was 52 years (range, 23 to 60 years) and all ten patients were Caucasian (100\%). These patients had received a median of two prior therapies in the metastatic setting. Five patients had an Eastern Cooperative Oncology Group (ECOG) performance status [15] of 0 and five patients had an ECOG of 1 . The patient characteristics are summarized in Table 1.

Nine of ten patients with $B R A F$ mutations were treated with BRAF inhibitors, either as a single agent (7 patients) or in combination with a MEK inhibitor (2 patients). The remaining one patient was treated with a single-agent MEK inhibitor. The clinical trials are summarized in Additional file 2. Four of ten patients (40\%) had a CR, 
Table 1 Demographics and clinical characteristics of 10 patients with BRAF mutation- positive melanoma

\begin{tabular}{|c|c|c|c|}
\hline Variable & Group & $\begin{array}{l}\text { No. of } \\
\text { patients } \\
(n=10)\end{array}$ & $\%$ \\
\hline \multirow[t]{4}{*}{ Age, years } & Median & 52 & \\
\hline & Range & $23-60$ & \\
\hline & $<50$ & 5 & 50 \\
\hline & $\geq 50$ & 5 & 50 \\
\hline \multirow[t]{2}{*}{ Sex } & Men & 7 & 70 \\
\hline & Women & 3 & 30 \\
\hline Race & Caucasian & 10 & 100 \\
\hline \multirow{4}{*}{$\begin{array}{l}\text { Number of prior } \\
\text { therapies }\end{array}$} & Median & 2 & \\
\hline & Range & $0-5$ & \\
\hline & $\leq 2$ & 8 & 80 \\
\hline & $>2$ & 2 & 20 \\
\hline \multirow[t]{2}{*}{ Lactate dehydrogenase $^{a}$} & supper limit of normal & 9 & 90 \\
\hline & > upper limit of normal & 1 & 10 \\
\hline \multirow[t]{2}{*}{ TNM stage M1c } & No & 6 & 60 \\
\hline & Yes & 4 & 40 \\
\hline \multirow[t]{2}{*}{ ECOG PS } & 0 & 5 & 50 \\
\hline & 1 & 5 & 50 \\
\hline \multirow[t]{3}{*}{ Therapy } & BRAF inhibitor alone & 7 & 70 \\
\hline & MEK inhibitor alone & 1 & 10 \\
\hline & $\begin{array}{l}\text { BRAF/MEK inhibitor } \\
\text { combination }\end{array}$ & 2 & 20 \\
\hline \multirow[t]{2}{*}{ Treatment response } & Complete response & 4 & 40 \\
\hline & Partial response & 6 & 60 \\
\hline
\end{tabular}

apper limit of normal in our institution $=618 \mathrm{IU} / \mathrm{L}$.

Abbreviations: ECOG Eastern Cooperative Oncology Group, PS Performance Status, TNM tumor, node, metastasis.

three of which are ongoing for about two years or longer $(\mathrm{TTF}=5.6,23.6+, 27.4+$, and $28.7+$ months). Six patients $(60 \%)$ attained a $\mathrm{PR}(\mathrm{TTF}=3.0,4.2,5.7,7.0$, 7.9, and 11.2 months) (Figures 1 and 2).

\section{Somatic genomic alterations}

Eight of ten patients had results of NGS analysis performed on tissues biopsied prior to treatment (range, 2 to 53 months) (Table 2). In two patients, NGS analyses were done on tissues biopsied after treatment initiation; in one case (case \#4), the tissue for NGS analysis was obtained six months after treatment initiation from a continuously progressive lesion; in another case (case \#5), NGS analyses was performed on three different tissue samples from progressive lesions (at 6, 9 and 26 months after treatment initiation).

All patients harbored a BRAF V600E mutation. One patient (case \#2) had an additional BRAF T599S mutation in exon 15 . Seven of ten patients (70\%) had somatic alterations of known significance in addition to $B R A F$ mutations (Table 2). In addition, seven out of ten patients $(70 \%)$ had somatic alterations of unclear significance (Additional file 3).

\section{Molecular alterations in responding patients}

Two of three patients (66\%) with long term CR (28.7+ and 23.6+ months) (cases \#1 and 3) had a BRAF mutation as the only molecular alteration of known significance and one of seven patients (14\%) with transient $\mathrm{CR} / \mathrm{PR}$ had only a BRAF mutation (case \#6). Alterations of known significance found in other patients included NRAS mutation ( $n=2$ patients), PTEN deletion $(n=1$ patient), adenomatous polyposis coli $(A P C)$ mutation ( $n=1$ patient), cyclin-dependent kinase inhibitor $(C D K N)$ $2 A$ or $2 B$ deletion ( $n=3$ patients), PAX5 deletion $(n=1$ patient), neurofibromin 1 (NF1) mutation ( $n=1$ patient), $B R A F$ amplification ( $n=2$ patients), Aurora kinase $A$ amplification ( $n=1), M E T$ amplification ( $n=1$ patient), microphthalmia-associated transcription factor (MITF) amplification ( $n=1$ patient), and, $\mathrm{v}$-myc avian myelocytomatosis viral oncogene homolog $(M Y C)$ amplification ( $n=1$ patient) (Table 2$)$. New mutations within the $B R A F$ gene itself were not observed except in one case (case \#2) who attained a prolonged CR and had a T599S in addition to a V600E mutation.

\section{Responses in patients with simultaneous NRAS mutations on treatment}

NRAS mutations (Q61R and Q61K in codon 61) were detected in two of ten patients (20\%). One patient (case \#4) attained a PR (TTF $=11.2$ months) and had an NRAS mutation on NGS analysis from tissue taken from a continuously progressing lesion 6 months after treatment initiation; adequate tissue sample for testing before treatment was not available. The other patient achieved a CR (case \#8; TTF = 5.6 months) and had an NRAS mutation detected in a single PCR assay from a biopsy of the solitary hepatic lesion that progressed after a brief period of initial response (3.8 months after treatment initiation; Figure 3), while the pre-treatment NGS analysis on this patient failed to show an NRAS mutation (17 months before treatment).

\section{Molecular evolution with progression}

Several patients had more than one biopsy performed, and molecular evolution was demonstrated in their results of molecular analysis. One patient with a CR (case \#8; TTF $=5.6$ months) had NGS analysis performed on a pre-treatment biopsy (17 months before treatment) that showed a BRAF and an NF1 mutation. Subsequently, a second biopsy 3.8 months after treatment initiation showed an NRAS mutation (Q61K) by single PCR-based analysis (Table 2). One patient (case \#5) had three different results on three different biopsy samples from 


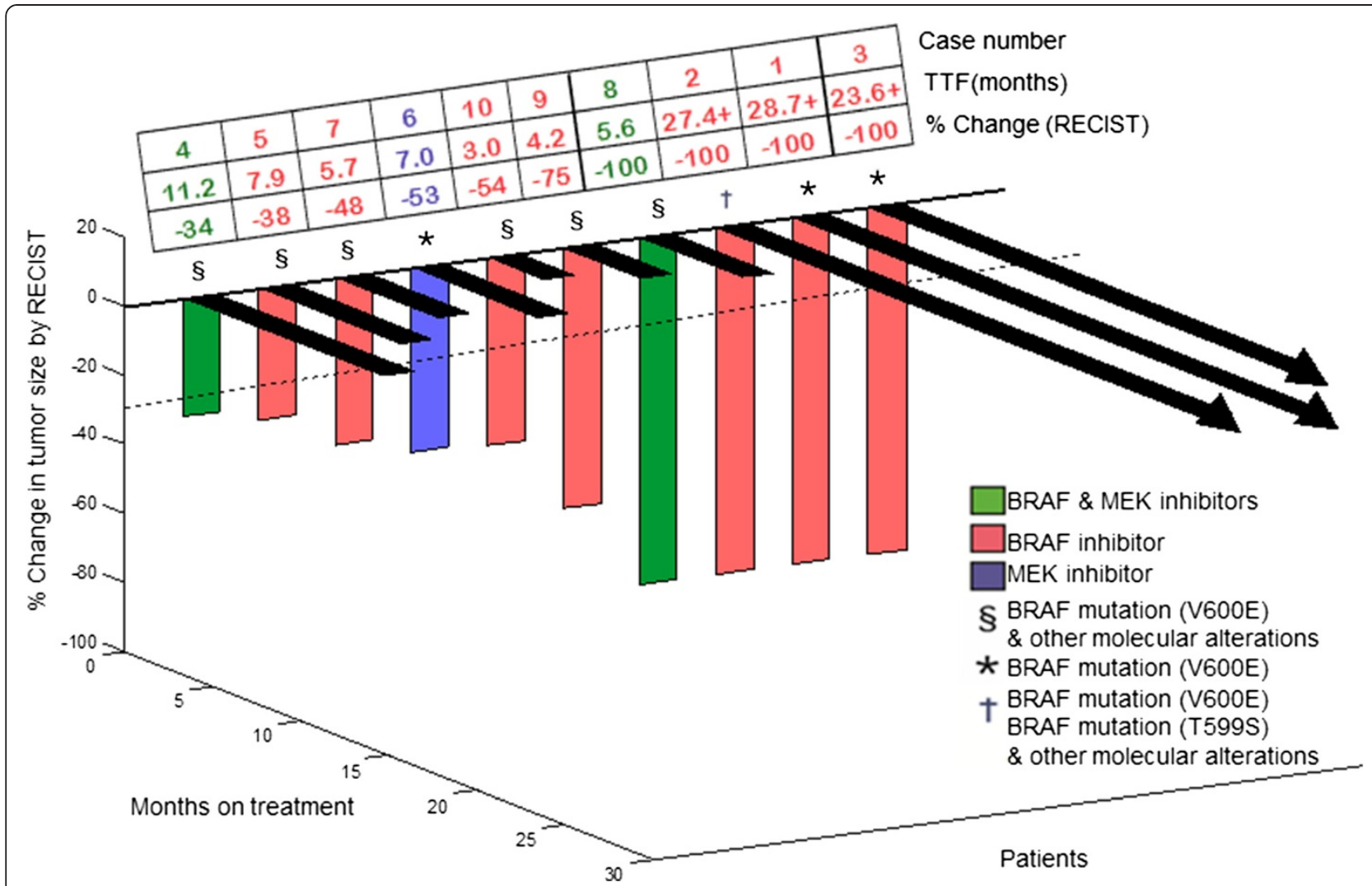

Figure 1 3-D waterfall plot. Best response by RECIST, of ten patients with BRAF-positive melanoma. Time to treatment failure in months is represented by solid lines and the arrow indicates that the patient was still on study when the data was censored.

progressive lesions $(6,9$ and 26 months after treatment initiation) by NGS analysis. The first, at 6 months after treatment initiation, showed $C D K N 2 A, C D K N 2 B$ and $P A X 5$ deletion (in addition to a $B R A F$ mutation), while the second at 9 months after treatment started, showed an additional NF1 truncation. A third NGS analysis obtained
26 months after treatment initiation demonstrated only alterations of unclear significance (Additional file 3). Of interest, this patient was having a mixed response to $B R A F$-targeted treatment at the time of the third biopsy. That biopsy, which no longer showed the BRAF mutation, was obtained from a tumor that was enlarging;

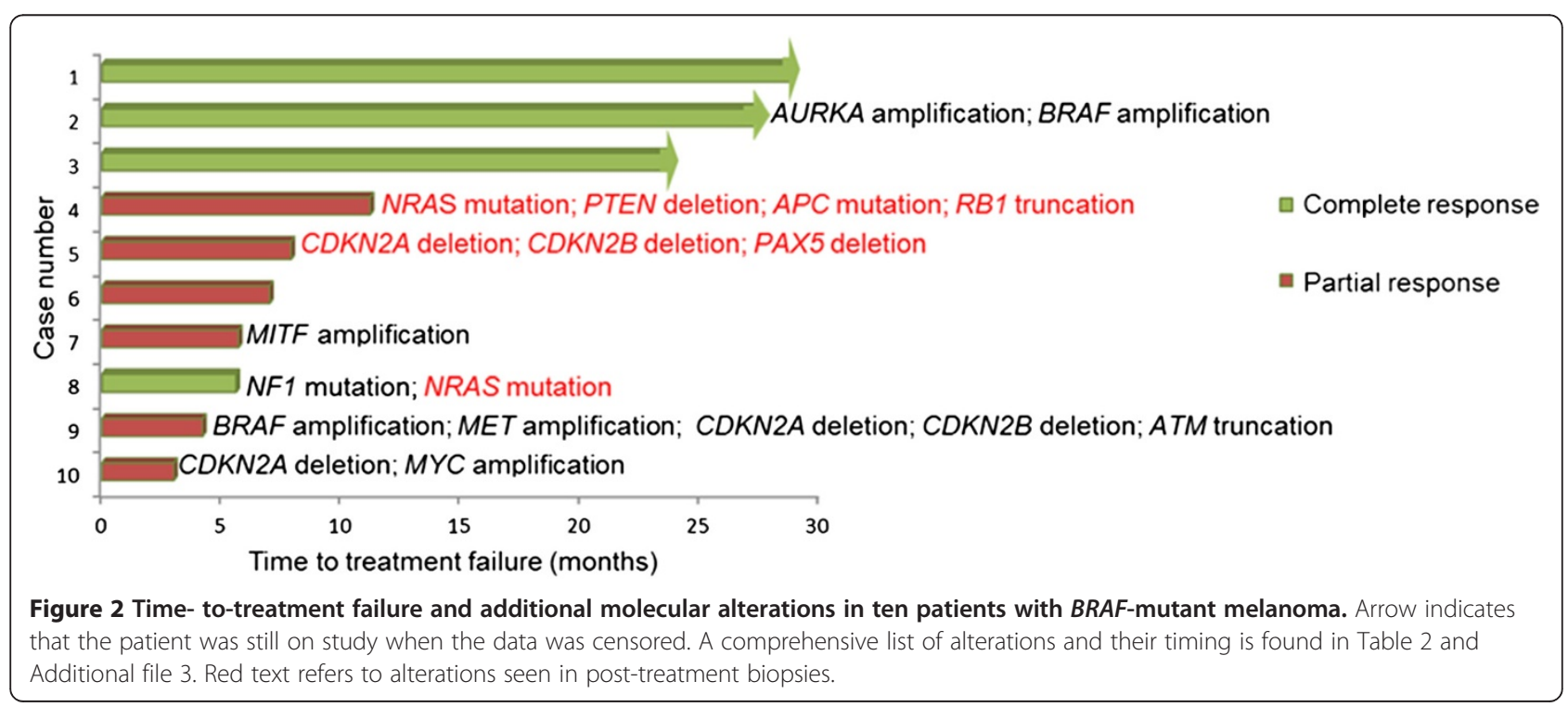


Table 2 NGS-based molecular alterations in BRAF-positive melanoma responders

\begin{tabular}{|c|c|c|c|c|c|c|c|c|c|}
\hline \multirow{2}{*}{$\begin{array}{l}\text { Case } \\
\text { no. }\end{array}$} & \multirow{2}{*}{$\begin{array}{l}\text { BRAF } \\
\text { mutation }\end{array}$} & \multirow{2}{*}{$\begin{array}{l}\text { Type of targeted } \\
\text { drug }\end{array}$} & \multirow{2}{*}{$\begin{array}{l}\text { Treatment } \\
\text { start date }\end{array}$} & \multirow{2}{*}{$\begin{array}{l}\text { Best } \\
\text { response (\%) }\end{array}$} & \multirow{2}{*}{$\begin{array}{l}\text { TTF } \\
\text { (months) }^{\mathrm{a}}\end{array}$} & \multirow{2}{*}{$\begin{array}{l}\text { Sampling date; time } \\
\text { from treatment }^{\mathrm{b}}\end{array}$} & \multicolumn{3}{|l|}{ Molecular analysis by NGS } \\
\hline & & & & & & & Mutations & Amplifications/deletions & Truncations \\
\hline 1 & V600E & BRAF inhibitor & 9/17/2009 & CR $(-100)$ & $28.7+$ & 5/1/2009; -4 months & BRAF (V600E) & None & None \\
\hline \multirow[t]{2}{*}{2} & V600E & \multirow[t]{2}{*}{ BRAF inhibitor } & \multirow[t]{2}{*}{ 10/7/2009 } & \multirow[t]{2}{*}{ CR $(-100)$} & \multirow[t]{2}{*}{$27.4+$} & \multirow[t]{2}{*}{ 1/22/2009; -9 months } & \multirow[t]{2}{*}{ BRAF (V600E); BRAF (T599S) } & \multirow{2}{*}{$\begin{array}{l}\text { AURKA amplification; BRAF } \\
\text { amplification }\end{array}$} & \multirow[t]{2}{*}{ None } \\
\hline & T599S & & & & & & & & \\
\hline 3 & V600E & BRAF inhibitor & $1 / 27 / 2010$ & CR $(-100)$ & $23.6+$ & 8/19/2005; -53 months & None $^{c}$ & None & None \\
\hline 4 & V600E & BRAF + MEK inhibitor & $11 / 11 / 2010$ & PR $(-34)$ & 11.2 & 5/17/2011; +6 months & $\begin{array}{l}\text { APC (R1171H); BRAF (V600E); } \\
\text { NRAS (Q61R) }\end{array}$ & PTEN deletion & RB1 truncation \\
\hline \multirow[t]{3}{*}{5} & V600E & BRAF inhibitor & $6 / 17 / 2009$ & PR $(-38)$ & 7.9 & $12 / 2 / 2009 ;+6$ months & BRAF (V600E) & $\begin{array}{l}\text { CDKN2A deletion; CDKN2B deletion; } \\
\text { PAX5 deletion }\end{array}$ & None \\
\hline & & & & & & 3/23/2010; +9 months & BRAF (V600E) & $\begin{array}{l}\text { CDKN2A deletion; CDKN2B deletion; } \\
\text { PAX5 deletion }\end{array}$ & NF1 truncation \\
\hline & & & & & & $8 / 24 / 2011 ;+26$ months & None & None & None \\
\hline 6 & V600E & MEK inhibitor & $4 / 1 / 2010$ & PR $(-53)$ & 7.0 & 9/17/2007; -31 months & BRAF (V600E) & None & None \\
\hline 7 & V600E & BRAF inhibitor & $1 / 12 / 2010$ & PR $(-48)$ & 5.7 & 11/5/2009; -2 months & BRAF (V600E) & MITF amplification & None \\
\hline 8 & V600E & BRAF + MEK inhibitor & $9 / 3 / 2010$ & $C R(-100)$ & 5.6 & 4/7/2009; -17 months & BRAF (V600E); NF1 (R 440*) & None & None \\
\hline 9 & V600E & BRAF inhibitor & $1 / 27 / 2010$ & $\mathrm{PR}(-75)$ & 4.2 & 11/11/2009; -2 months & BRAF (V600E) & $\begin{array}{l}\text { BRAF amplification; MET amplification; } \\
\text { CDKN2A deletion; CDKN2B deletion }\end{array}$ & ATM truncation \\
\hline 10 & V600E & BRAF inhibitor & $4 / 15 / 2010$ & PR $(-54)$ & 3.0 & 12/11/2009; -4 months & BRAF (V600E) & MYC amplification; CDKN2A deletion & None \\
\hline
\end{tabular}

${ }^{a}{ }^{\prime}+{ }^{\prime}=$ continuing on the study when data was censored.

${ }^{b}{ }^{\prime}+{ }^{\prime}=$ number of months biopsy was taken after treatment; ' - ' = number of months biopsy preceded treatment.

${ }^{c}=$ tissue sample obtained on 10/29/2008 was BRAF mutation-positive (V600E) by single PCR assay (case \#3).

$\mathrm{d}=$ tissue sample obtained from a progressive hepatic lesion on 12/27/2010 had an NRAS mutation (Q61K) in codon 61 by single PCR assay (case \#8).

Abbreviations: $C R$ Complete response, NGS Next generation sequencing, $P R$ Partial response, $T F$ Time to treatment failure. 

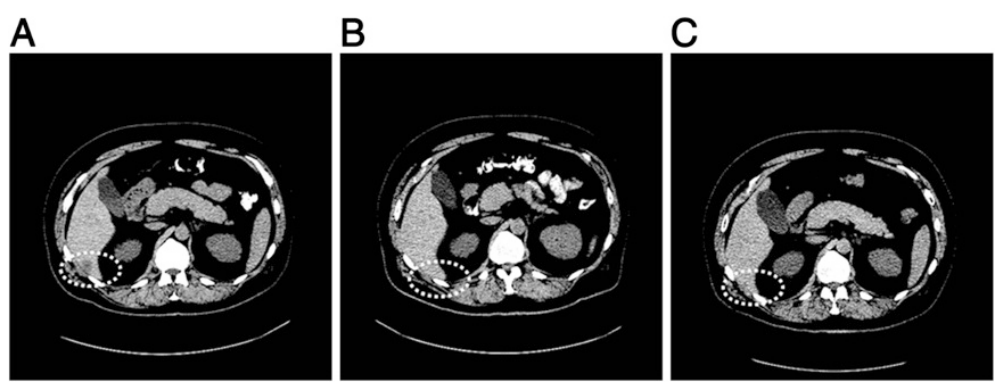

Figure 3 Computed tomography scans. A) at baseline, B) 2 months, and, C) 3.8 months after treatment initiation with a combination of a MEK and BRAF inhibitor, of a patient (case \#8) with short-lived CR (TTF = 5.6 months), who demonstrated an NRAS mutation in a biopsy obtained 3.8 months after treatment initiation. At 2 months, the liver lesion has regressed; at 3.8 months it recurred.

other tumors that had shown the BRAF mutation were regressing on $B R A F$-targeted therapy.

\section{Timing of molecular analysis}

One patient with an ongoing CR of 23.6+ months duration (case \#3) had NGS analysis performed on a biopsy obtained 53 months prior to treatment; no alterations of known significance were demonstrated. A second biopsy performed 15 months prior to treatment showed a V600E $B R A F$ mutation in a single PCR-based assay. This patient illustrates a phenomenon of interest, i.e., patients without $B R A F$ mutations can acquire the mutation with progression, and they can be sensitive to cognate targeted inhibitors.

Another patient (case \#6) with a biopsy 31 months prior to treatment had only a $B R A F$ mutation among the alterations of known significance. This patient achieved a PR lasting 7.0 months. All other patients with biopsies performed 2 to 17 months prior to treatment had additional alterations of known significance, except for one individual (case \#1) who had only a BRAF mutation and achieved a CR that is ongoing at $28.7+$ months.

\section{Discussion}

In order to better understand response and resistance, we analyzed patients with $B R A F$-mutant, advanced melanoma who achieved a PR or CR on a BRAF and/or MEK targeted agent. Previous reports [16] provide proof of principal for this next generation sequencing (NGS) platform. NGS identified a broad range of molecular alterations (Table 2; Additional file 3).

Most patients $(n=7)$ had multiple additional alterations of known oncogenic significance (Table 2). Pathways commonly affected, either directly or indirectly, included the PI3K/AKT/mammalian target of rapamycin (mTOR) axis (via PTEN alterations), the MAPK pathway (via NRAS mutations or NF1 alterations), as well as tumor suppressor signals (via $C D K N 2 A$ or $C D K N 2 B$ deletion or $R B 1$ truncation). Other abnormalities included deletion in $P A X 5$ (a paired box transcription factor), and amplifications in MET, BRAF and aurora kinase $A$. The adenomatous polyposis coli $(A P C)$ gene responsible for familial adenomatous polyposis, was mutated in one patient; this gene, when aberrant, activates the Wnt signaling pathway and induces chromosomal instability $[17,18]$. Loss of its function also triggers the adenomacarcinoma transition in colorectal cancer [19]. Additional mutations within the $B R A F$ gene itself were not observed except for patient \#2 who attained a prolonged CR and had a T599S in addition to a V600E mutation. BRAF amplification was seen in two patients.

These additional molecular abnormalities were at times evident long before treatment including, in one case, 17 months prior to starting targeted therapy (case \#8). Only three patients (cases \#1, 3 and 6) had no additional alterations of known significance as discerned by NGS, and two of these individuals achieved a prolonged CR with response ongoing $23.6+$ months (case \#3) and $28.7+$ months (case $\# 1$ ), respectively, after starting treatment with a BRAF inhibitor. The third patient (case \#6) achieved a transient PR (TTF $=7$ months). In case \#1, the tissue sample was obtained only 4 months prior to treatment. However, in cases 3 and 6, the tissue samples were acquired 53 and 31 months, respectively, prior to treatment; it is therefore unclear whether or not other biologically-significant alterations might have emerged closer to the time of treatment. One additional patient with a prolonged CR (TTF $=27.4+$ months; case \#2) demonstrated BRAF and aurora kinase $A$ amplification in addition to a $B R A F$ mutation in a tissue sample obtained 9 months before treatment.

Several observations herein warrant further exploration. First, it is conceivable that NGS analysis may reveal a subset of advanced disease that can achieve prolonged CR on a BRAF inhibitor alone. It is plausible that these patients are the ones that have only a $B R A F$ alteration, or only alterations that influence a redundant signal. For most patients with melanoma, the emergence of resistance is expected. It is somewhat surprising that certain individuals can achieve a CR while harboring numerous potential 
driving alterations. For instance, the patient with a CR of $27.4+$ months (case \#2) had an NGS profile demonstrating several possible driver alterations (BRAF and aurora kinase $A$ amplification) in addition to a $B R A F$ mutation. Amplifications in these genes are known to confer resistance to treatment with BRAF inhibitors, though it has been suggested that the resistance driven by $B R A F$ amplification can be overcome by higher doses of a BRAF inhibitor [20] or by combining MEK and BRAF inhibition [21]. The concept of oncogenic addiction may explain response in such individuals [22].

NGS analysis may also provide information that can be exploited to devise optimal combinations of targeted agents for patients at the time of their initial therapy or when resistance emerges. One patient who achieved a $\mathrm{CR}$, albeit of short duration (TTF $=5.6$ months; case \#8), may have done so because he was treated with a combination of a MEK and BRAF inhibitor. NGS revealed a mutation in NF1 in addition to a V600E BRAF mutation. $N F 1$ is an upstream suppressor that can modulate MEK signaling [23]. Such alterations confirm the need for strategies that incorporate combinations including BRAF and MEK inhibitors. In this patient, an NRAS mutation (Q61K) emerged 3.8 months after treatment initiation, which was demonstrated by a single PCR assay of the tissue sampled from the solitary liver lesion that progressed after treatment. This might have accounted for the patient's relapse. The second patient (case \#4) treated with a MEK and BRAF inhibitor combination demonstrated remarkable response ( $-34 \%$; PR); a cystic lesion in the chest wall eventually showed progression. Fine needle aspiration cytology of the lesion revealed a Q61R mutation in the NRAS gene and other molecular aberrations in addition to the BRAF mutation.

Altogether two patients developed NRAS mutations after treatment (Figure 2) (cases \#4 and \#8). The emergence of an NRAS mutation is known to confer resistance to BRAF inhibitors [7], and may therefore explain the failure to achieve a durable response in these patients $(\mathrm{n}=1$ with PR and $\mathrm{n}=1$ with transient CR) [24,25]. The presence of an NRAS mutation in addition to a BRAF mutation in patients treated with BRAF inhibitors may reactivate the MAP kinase (MAPK) pathway through CRAF [7]. Although MEK inhibitors might seem a rational choice for patients bearing NRAS alterations as anti-tumor activity has been reported in NRAS-mutant cutaneous melanoma patients on MEK 162 [26], experience suggests limited efficacy in such patients with other MEK inhibitors [27]. It is unclear whether this is due to the fact that NRAS signaling is incompletely extinguished by MEK inhibitors or if these tumors bear additional alterations that confer resistance.

One patient had a PTEN deletion (case \#4). Alterations in the $\mathrm{PI} 3 \mathrm{~K} / \mathrm{AKT} / \mathrm{mTOR}$ pathway have been shown to be operative in multiple tumors including melanoma [28]. For instance, PTEN loss is found in $30-50 \%$ of melanomas [29], PIK3CA mutation in 3\% [30], and changes in AKT expression in some melanomas [31]. These are actionable alterations in that multiple PIK3CA, AKT and mTOR inhibitors are in clinical trials or are already approved.

Recently, molecular evolution with progression and to some degree heterogeneity between tumors in individual patients has been described [32]. For instance, Wilmott and colleagues [33] reported different subclones in tumor tissue from a single metastatic site in a $B R A F$-mutant melanoma patient, following progression of disease after seven months of treatment with the BRAF inhibitor vemurafenib; one clone had an additional NRAS mutation. Our data supports such heterogeneity and demonstrates the role that advanced molecular technology may play in understanding and addressing mixed responses. For instance, a patient (case \#5) with a PR for 7.9 months had three different biopsies from progressive lesions performed at 6, 9 and 26 months after treatment initiation. Each of these biopsies was analyzed using NGS and each demonstrated different results. The first two analyses, at 6 and 9 months, showed CDKN2A, CDKN2B and PAX5 deletions, but the sample obtained 9 month after treatment initiation also showed an NF1 truncation, probably indicating accumulation of additional changes with disease progression. The results from a sample obtained 26 months after treatment initiation, however, showed no BRAF mutation, or other molecular alterations of known significance. Of interest, the latter biopsy was taken from a tumor that was increasing in size on a BRAF inhibitor, while the other tumors had regressed.

There were several important limitations of this study. First, it is a retrospective study and as such tissue samples were not collected at a uniform time. Second the small number of patients in this pilot study precludes statistical assessments. Third, we focused on responders. Data on non-responders may be equally informative in identifying the mechanisms that confer resistance.

This study demonstrates multiple additional molecular alterations amongst $B R A F$ mutation-positive patients with advanced melanoma who achieved a CR or PR on BRAF targeted agents. These alterations may be responsible for the development of resistance to treatment with BRAF and/or MEK inhibitors. We did not observe frequent new mutations in the $B R A F$ gene itself correlating with resistance, consistent with the reported stability of the BRAF mutation in melanoma [34]; unlike the situation with other kinases such as $B C R-A B L$ or epidermal growth factor receptor (EGFR), where resistance is often mediated by acquisition of additional mutations within the same gene. Given that some $B R A F$-mutant melanoma patients fail to respond to BRAF inhibitors, and that the majority of patients who do respond to BRAF/MEK inhibitors 
eventually develop resistance, there is an urgent need to identify possible combination treatments that may be effective. Of interest, some patients with advanced melanoma can achieve prolonged $\mathrm{CR}$, and our investigation suggests that some of these individuals may harbor only a $B R A F$ mutation despite the advanced state of their disease. Further, patients can achieve CR while still harboring aberrations other than those targeted by the agent given. (In our study, 2 of the 4 patients who achieved CRs harbored additional aberrations: aurora kinase and BRAF amplification in one patient, and an NF1 and NRAS mutation in one patient). Therefore tumor complexity does not preclude a complete response. Finally, while obtaining biopsies close to the time of treatment may be ideal, our study illustrates that data obtained from biopsies distant to the time of treatment may still demonstrate aberrations that could be responsible for response and/or resistance. A concern that has been raised is whether patients with multiple aberrations can respond to targeted treatment of one of those aberrations, or whether such treatment is futile.

\section{Conclusion}

Our study demonstrates that most of our excellent responders had multiple aberrations. Having these aberrations may (or may not) preclude "cure", but they do not preclude an excellent response. Our study suggests that further investigation of NGS for identification of actionable molecular alterations before treatment and at the time of resistance is warranted.

\section{Additional files}

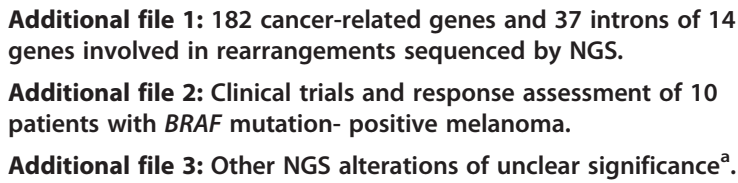

\section{Abbreviations}

PR: Partial response; CLIA: Certified molecular diagnostic laboratory; CR: Complete response; DNA: Deoxyribonucleic acid; ECOG: Eastern Cooperative Oncology Group; NGS: Next generation sequencing; PCR: Polymerase chain reaction; RECIST: Response evaluation criteria in solid tumors; TTF: Time to treatment failure.

\section{Competing interests}

RY, PS and, MC are employees and stock holders in Foundation Medicine Inc. GF received research funding and travel reimbursement for ESMO 2010 from Glaxo Smithkline. KK received honoraria from Genentech and served as consultant to Roche and Genentech. RK received research funding from Hoffman-LaRoche and Genentech. All remaining authors have declared no conflicts of interest.

\section{Authors' contributions}

JW, RY, PH, PS, MC and RK designed the study and analyzed the results. JW performed the literature search; JW, GF, AT, and, DH collected the data. Data was analyzed by JW, RY, PS, MC and RK and interpreted by JW, RY, GF, KK, AT, PS, DH, MC and RK. All authors contributed to writing. All authors reviewed and approved the final manuscript.

\section{Acknowledgments}

Support was provided in part by a grant from Foundation Medicine. We thank the patients and their families. The authors thank Hemwattie Ramnauth, and, Bettzy Stephen in the Department of Investigational Cancer Therapeutics at MD Anderson Cancer Center for their role in data collection.

\section{Author details}

'Department of Investigational Cancer Therapeutics - a Phase I Clinical Trials Program, Unit 455, The University of Texas MD Anderson Cancer Center, 1515 Holcombe Blvd, Houston, TX 77030, USA. ${ }^{2}$ Foundation Medicine, 150 Second Street, Cambridge, MA 02141, USA. ${ }^{3}$ Department of Melanoma Medical Oncology, The University of Texas MD Anderson Cancer Center 1515 Holcombe Blvd, Houston, TX, 77030, USA. ${ }^{4}$ Center for Personalized Cancer Therapy, Moores Cancer Center, The University of California San Diego, 3855 Health Sciences Drive, La Jolla, CA 92093, USA.

Received: 30 May 2014 Accepted: 20 January 2015

Published online: 18 February 2015

\section{References}

1. Davies H, Bignell GR, Cox C, Stephens P, Edkins S, Clegg S, et al. Mutations of the BRAF gene in human cancer. Nature. 2002;417(6892):949-54.

2. Sosman JA, Kim KB, Schuchter L, Gonzalez R, Pavlick AC, Weber JS, et al. Survival in BRAF V600-mutant advanced melanoma treated with vemurafenib. N Engl J Med. 2012;366(8):707-14.

3. Chapman PB, Hauschild A, Robert C, Haanen JB, Ascierto P, Larkin J, et al Improved survival with vemurafenib in melanoma with BRAF V600E mutation. N Engl J Med. 2011;364(26):2507-16.

4. Falchook GS, Long GV, Kurzrock R, Kim KB, Arkenau TH, Brown MP, et al. Dabrafenib in patients with melanoma, untreated brain metastases, and other solid tumours: a phase 1 dose-escalation trial. Lancet. 2012;379(9829):1893-901.

5. Villanueva J, Vultur A, Lee JT, Somasundaram R, Fukunaga-Kalabis M, Cipolla AK, et al. Acquired resistance to BRAF inhibitors mediated by a RAF kinase switch in melanoma can be overcome by cotargeting MEK and IGF-1R/PI3K. Cancer Cell. 2010;18(6):683-95.

6. Sullivan RJ, Flaherty KT. BRAF in melanoma: pathogenesis, diagnosis, inhibition, and resistance. J Skin Cancer. 2011;2011:423239.

7. Nazarian R, Shi H, Wang Q, Kong X, Koya RC, Lee H, et al. Melanomas acquire resistance to $B-R A F(V 600 E)$ inhibition by RTK or N-RAS upregulation. Nature. 2010;468(7326):973-7.

8. Infante JR, Falchook GS, Lawrence DP, Weber JS, Kefford RF, Bendell JC, et al. Phase I/II study to assess safety, pharmacokinetics, and efficacy of the oral MEK 1/2 inhibitor GSK1120212 (GSK212) dosed in combination with the oral BRAF inhibitor GSK2118436 (GSK436). In: J Clin Oncol 29: 2011 (suppl; abstr CRA8503): 2011. 2011.

9. Zuo Z, Chen SS, Chandra PK, Galbincea JM, Soape M, Doan S, et al. Application of COLD-PCR for improved detection of KRAS mutations in clinical samples. Mod Pathol. 2009;22(8):1023-31.

10. Nosho K, Kawasaki T, Ohnishi M, Suemoto Y, Kirkner GJ, Zepf D, et al. PIK3CA mutation in colorectal cancer: relationship with genetic and epigenetic alterations. Neoplasia. 2008;10(6):534-41.

11. Sakr RA, Barbashina V, Morrogh M, Chandarlapaty S, Andrade VP, Arroyo CD, et al. Protocol for PTEN expression by immunohistochemistry in formalinfixed paraffin-embedded human breast carcinoma. Appl Immunohistochem Mol Morphol. 2010;18(4):371-4.

12. Beltran $H$, Yelensky R, Frampton GM, Park K, Downing SR, Macdonald TY, et al. Targeted next-generation sequencing of advanced prostate cancer identifies potential therapeutic targets and disease heterogeneity. Eur Urol. 2013;63(5):920-6

13. Therasse P, Arbuck SG, Eisenhauer EA, Wanders J, Kaplan RS, Rubinstein L, et al. New guidelines to evaluate the response to treatment in solid tumors. European Organization for Research and Treatment of Cancer, National Cancer Institute of the United States, National Cancer Institute of Canada. J Natl Cancer Inst. 2000;92(3):205-16.

14. Eisenhauer EA, Therasse $P$, Bogaerts J, Schwartz LH, Sargent D, Ford R, et al. New response evaluation criteria in solid tumours: revised RECIST guideline (version 1.1). Eur J Cancer. 2009:45(2):228-47.

15. Oken MM, Creech RH, Tormey DC, Horton J, Davis TE, McFadden ET, et al. Toxicity and response criteria of the Eastern Cooperative Oncology Group. Am J Clin Oncol. 1982;5(6):649-55. 
16. Lipson D, Capelletti M, Yelensky R, Otto G, Parker A, Jarosz M, et al. Identification of new ALK and RET gene fusions from colorectal and lung cancer biopsies. Nat Med. 2012;18(3):382-4.

17. Groden J, Thliveris A, Samowitz W, Carlson M, Gelbert L, Albertsen H, et al. Identification and characterization of the familial adenomatous polyposis coli gene. Cell. 1991;66(3):589-600.

18. Rother J, Jones D. Molecular markers of tumor progression in melanoma. Current genomics. 2009;10(4):231-9.

19. Ceol CJ, Pellman D, Zon LI. APC and colon cancer: two hits for one. Nat Med. 2007;13(11):1286-7.

20. Shi H, Moriceau G, Kong X, Lee MK, Lee $H$, Koya RC, et al. Melanoma whole-exome sequencing identifies (V600E) B-RAF amplification-mediated acquired B-RAF inhibitor resistance. Nat Commun. 2012;3:724.

21. Corcoran RB, Dias-Santagata D, Bergethon K, lafrate AJ, Settleman J, Engelman JA. BRAF gene amplification can promote acquired resistance to MEK inhibitors in cancer cells harboring the BRAF V600E mutation. Sci Signal. 2010;3(149):ra84.

22. Weinstein IB. Cancer: addiction to oncogenes-the Achilles heal of cancer. Science. 2002;297(5578):63-4.

23. Pratilas CA, Solit DB. Targeting the mitogen-activated protein kinase pathway: physiological feedback and drug response. Clin Cancer Res. 2010;16(13):3329-34

24. Flaherty KT, Fisher DE. New strategies in metastatic melanoma: oncogenedefined taxonomy leads to therapeutic advances. Clin Cancer Res. 2011;17(15):4922-8.

25. Flaherty KT, Hodi FS, Fisher DE. From genes to drugs: targeted strategies for melanoma. Nat Rev Cancer. 2012;12(5):349-61.

26. Ascierto PA, Berking C, Agarwala SS, Schadendorf D, Van Herpen C, Queirolo $P$, et al. Efficacy and safety of oral MEK162 in patients with locally advanced and unresectable or metastatic cutaneous melanoma harboring BRAF V600 or NRAS mutations. In: J Clin Oncol 30, 2012 (suppl; abstr 8511): 2012. 2012.

27. Falchook GS, Lewis KD, Infante JR, Gordon MS, Vogelzang NJ, DeMarini DJ, et al. Activity of the oral MEK inhibitor trametinib in patients with advanced melanoma: a phase 1 dose-escalation trial. The Lancet Oncology. 2012;13(4):782-9.

28. Bartholomeusz C, Gonzalez-Angulo AM. Targeting the PI3K signaling pathway in cancer therapy. Expert Opin Ther Targets. 2012;16(1):121-30.

29. Wu H, Goel V, Haluska FG. PTEN signaling pathways in melanoma. Oncogene. 2003;22(20):3113-22.

30. Omholt K, Krockel D, Ringborg U, Hansson J. Mutations of PIK3CA are rare in cutaneous melanoma. Melanoma Res. 2006;16(2):197-200.

31. Stahl JM, Sharma A, Cheung M, Zimmerman M, Cheng JQ, Bosenberg MW, et al. Deregulated Akt3 activity promotes development of malignant melanoma. Cancer Res. 2004;64(19):7002-10.

32. Gerlinger M, Rowan AJ, Horswell S, Larkin J, Endesfelder D, Gronroos E, et al. Intratumor heterogeneity and branched evolution revealed by multiregion sequencing. N Engl J Med. 2012;366(10):883-92.

33. Wilmott JS, Tembe V, Howle JR, Sharma R, Thompson JF, Rizos $\mathrm{H}$, et al. Intratumoral molecular heterogeneity in a BRAF-mutant, BRAF inhibitorresistant melanoma: a case illustrating the challenges for personalized medicine. Mol Cancer Ther. 2012;11(12):2704-8,

34. Sigalotti L, Fratta E, Parisi G, Coral S, Maio M. Stability of BRAF V600E mutation in metastatic melanoma: new insights for therapeutic success? Br J Cancer. 2011;105(2):327-8.

\section{Submit your next manuscript to BioMed Central and take full advantage of:}

- Convenient online submission

- Thorough peer review

- No space constraints or color figure charges

- Immediate publication on acceptance

- Inclusion in PubMed, CAS, Scopus and Google Scholar

- Research which is freely available for redistribution 